\title{
Bespoke payment plans to secure and grow your patient base
}

If you have seen the increased patient demand for new services like facial aesthetics, or teeth-straightening into your practice, have you considered the flexible payment platform from Simplyhealth Professionals? The platform helps you to increase treatment income and acceptance. You can use the platform to create a bespoke payment plan for each patient to spread the cost of their treatment and make it easier for patients to take up treatment plans.

The platform also includes a simple online application form and reporting suite, so you are always up to date with patient applications and if patients' payments are up to date.

With increasing demand from patients for orthodontic and aesthetic treatments, our platform can help you remove barriers to treatment acceptance and make treatment plans more affordable and convenient in your practice.

A payment plan using our flexible payment platform provides a way for patients to spread the costs of one off and short course elective treatments over a period of three to ten months. You decide how much your patients pay and how long the treatment will last, whilst your patients benefit from regular manageable payments to feel happy with their smile.

In addition, you can also use the flexible payment platform to spread the cost of ongoing dental treatment plans for your patients with a simple monthly subscription payment. This works well for treatments including, additional private hygiene, dermal fillers, anti-wrinkle, implant maintenance and teeth-whitening.

\section{How does it work?}

- You can create, for example, a teeth-straightening plan online in minutes, there are no paper forms to complete and send

- There is a dedicated support team to help you with any on boarding when you start in addition to on demand webinar training

- You create your own literature through our online design portal

- You can tailor the amount and number of monthly payments to each patient depending on their treatment type.

\section{What are the key benefits?}

- You are in control of the plan, you can take a deposit up front and decide the level of affordable monthly payment with the patient

- You have access to online, real-time reports - you won't have to wait to find out if a patient has paid

- Patients can sign up instantly in practice

- Increased patient retention

- No credit checks needed for the patient

- No credit licence is required by the practice to use the flexible payment platform

- Encourages long term treatment planning and attendance patients won't have to save up for treatments or skip appointments.

Angela Watson, Practice Manager and Dental Nurse at Cramlington Dental Centre has recently started using the

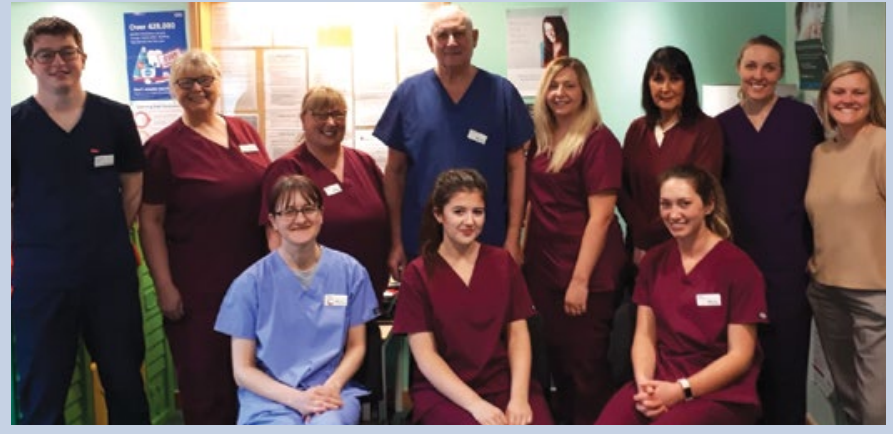

Cramlington Dental Centre Dental Team, (far right) Angela Watson, Practice Manager and Dental Nurse at Cramlington Dental Centre

Simplyhealth Professionals flexible payment platform for both teeth-whitening and orthodontic services in the practice:

'We've had a lot of enquiries about straightening techniques and teeth-whitening since we started to offer them, though this is not a particularly affluent area and for some patients these treatments have always been simply out of reach due to the cost. We could see that many wanted to consider such treatments and we were keen to find a viable means of spreading the cost for them.

'The team at Simplyhealth Professionals really helped us to understand the flexible payment platform and were able to make us feel confident about suggesting plans to patients. We proposed it initially just for teeth-whitening, which currently costs $£ 250$ here. Many of our patients would find $£ 250$ a substantial outlay, so as much as they wanted to go ahead with it, they'd often put it on hold.

'But when we worked out a plan for them that meant they only needed to pay $£ 50$ up front then $£ 20$ a month for the ten months following the treatment, it suddenly became far more feasible and we've had a really good take-up. Patients have been so pleased with the results, it's a great treatment to boost people's self-confidence.

'The reassuring thing is that because we're in control of the terms of the plans ourselves, we can calculate how much we need to receive as an up-front payment to cover lab fees and materials, then the rest is received monthly, so there's very little risk involved. And it only costs the practice $£ 1$ per patient ${ }^{\star}$ per month for Simplyhealth Professionals to manage collection of the payments, so there's hardly any effort on our part. What's more, we can access each patient's payment report at any time through the online portal, so we can always check that payments are being made.

'We produce a bespoke printed statement for each patient, detailing exactly what they will need to pay up front and the precise amount of each monthly payment for whatever term we agree between us - from three to ten months. They've been really successful for us, our patients love them and they're a great asset for the practice.'

*Payments are collected from the patient and passed on to the practice, less the payment collection fee of $£ 1.00$ including VAT.

To find out more, or to set up a plan, call 08001699962 or visit www.denplan.co.uk/fpp. Please note: The flexible payment platform is unavailable in the Channel Islands and Isle of Man. 